\section{Principales causas de automedicación en estudiantes del área de la salud}

\author{
Main causes of self-medication among students in the health area
}

Adela Alba Leonel,* Samantha Papaqui Alba, Fernando Castillo Nájera, Jorge Ricardo Medina Torres," Joaquín Papaqui Hernández,? Roberto Sánchez Ahedo**

\section{RESUMEN}

Introducción: La OMS identificó a la automedicación como un componente del autocuidado en la salud; $80 \%$ de la población mexicana usa medicamentos por iniciativa propia sin indicación médica y sin receta. Objetivo: Identificar las principales causas de automedicación en estudiantes del área de la salud. Material y métodos: Se realizó un estudio transversal donde el universo del estudio fueron estudiantes del área de la salud de nivel licenciatura y postgrado. Se utilizó un instrumento exprofeso de automedicación. Resultados: La muestra fue de 305 estudiantes, 72\% correspondió al género femenino, $71 \%$ tiene entre 17 a 30 años. La prevalencia de automedicación fue del 88\%. Las razones principales por la que se automedican son por falta de tiempo 42.1\%, fácil acceso a los medicamentos 37.4\% y $14.4 \%$ por economía. Y el principal motivo de salud por que se automedican es por algias 73.8\%, gripe 49.7\%, fiebre $33.8 \%$, gastroenteritis $27.8 \%$ y edema $26.4 \%$. Conclusiones: La automedicación es frecuente en estudiantes del área de la salud y las principales causas de automedicación son falta de tiempo, fácil acceso y los motivos son: algias, gripe y fiebre. El profesional de salud debe establecer estrategias de prevención para evitar el enmascaramiento y agravar el proceso de la enfermedad, resistencia a medicamentos, intoxicación e inclusive la muerte. Asimismo, debe fomentar que la automedicación es un componente del autocuidado.

Palabras clave: Automedicación, autocuidado, estudiantes del área de la salud.

\begin{abstract}
Introduction: WHO identified self-medication as a component of self-care in health; $80 \%$ of the Mexican population uses medicines on their own initiative without medical advice and without a prescription. Objective: To identify the main causes of self-medication among students in the health area. Material and methods: A cross-sectional study was carried out where the universe of the study was students of the health area of undergraduate and postgraduate level. An ex profeso self-medication instrument was used. Results: The sample was of 305 students, $72 \%$ corresponded to the female gender, $71 \%$ are between 17 and 30 years old. The prevalence of self-medication was $88 \%$. The main reasons why they self-medicate are the lack of time $42.1 \%$, easy access to medicines $37.4 \%$ and $14.4 \%$ for economy. And the main reason for self-medication is ache $73.8 \%$, flu $49.7 \%$, fever $33.8 \%$, gastroenteritis $27.8 \%$ and edema $26.4 \%$. Conclusions: Self-medication is frequent among students in the health area and the main causes of self-medication are lack of time, easy access and the reasons are: pain, flu and fever. The health professional must establish prevention strategies to avoid masking and aggravate the disease process, drug resistance, intoxication and even death. It should also encourage that self-medication is a component of self-care.
\end{abstract}

Keywords: Self-medication, self-care, students of the health area.

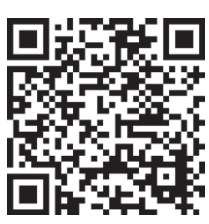

* Doctora en Ciencias de la Salud campo disciplinar Epidemiología. Profesor de carrera Asociado C, Escuela Nacional de Enfermería y Obstetricia, UNAM.

‡Estudiante de Medicina

St. Luke Escuela de

Medicina.

${ }^{5}$ Maestro en

Administración de Hospitales. Jefe de Unidad Médica en los Servicios de Salud Pública de la Ciudad de México y Profesor de asignatura de la Facultad de Medicina, UNAM.

" Licenciado en

Enfermería, Escuela

Nacional de Enfermería y

Obstetricia, UNAM.

" Ingeniero en Informática y Especialista en Estadística Aplicada, Instituto Mexicano del Seguro Social.

** Médico Especialista en Medicina Familiar, Profesor de asignatura de Cirugía de la Facultad de Medicina, UNAM.

Correspondencia: AAL, adelaalbaleonel65@ gmail.com Conflicto de intereses: Los autores declaran que no tienen.

Citar como: Alba LA, Papaqui AS, Castillo N F, Medina TJR, Papaqui $H J$, Sánchez AR. Principales causas de automedicación en estudiantes del área de la salud. Rev CONAMED 2019; 24(3): 3-9. doi: 10.35366/92889

Financiamiento: Ninguno.

Recibido: 25/01/2019.

Aceptado: 25/06/2019. 


\section{INTRODUCCIÓN}

La Organización Mundial de la Salud (OMS) define a la automedicación como la libertad de cada persona para consumir medicamentos disponibles para la venta sin prescripción médica, para el tratamiento de enfermedades o síntomas reconocidos por el paciente mismo. Así mismo en su línea estratégica para todos en el año 2000 consideró a los «autocuidados responsables» como uno de los principales ejes para la promoción de la salud. El término autocuidado incluye la automedicación, el autotratamiento y los primeros auxilios. La automedicación la identificó como un componente del autocuidado en la salud. ${ }^{1-3}$

Otra definición es cuando el usuario trata sus enfermedades o síntomas con medicamentos que han sido probados y están disponibles para la venta sin prescripción y que son seguros y eficaces cuando se usan de acuerdo con las condiciones establecidas.

Actualmente la automedicación se ha convertido en un problema de salud pública, ya que su práctica cada vez es más frecuente en la población a nivel mundial. Asimismo, este fenómeno se ha considerado como una forma de autocuidado, el cual puede tener un impacto positivo en los sistemas de salud en cuanto a la reducción de gastos, menor demanda en la asistencia por problemas como algias; sin embargo, también puede tener un gran impacto negativo, ya que puede enmascarar y agravar el proceso de la enfermedad o bien que se emita un diagnóstico y tratamiento inadecuado, interacciones medicamentosas y la posibilidad de presentarse reacciones adversas a los medicamentos (RAM). ${ }^{4}$ La OMS, considera que la automedicación es un factor importante en las RAM.

Algunos estudios del siglo pasado, realizados en España, Sudáfrica y EUA muestran que entre 40 y $90 \%$ de episodios enfermizos fueron tratados mediante el autocuidado., 5

Cabe mencionar que esta práctica se presenta cada día más en países en vías de desarrollo y quizás uno de los factores que intervienen en la automedicación tengan que ver con la falta de accesibilidad a los servicios de salud, demoras en la atención, falta de seguridad social, facilidad en la venta de medicamentos, nivel de ingresos económicos, acceso de información de los medicamentos por diferentes medios de comunicación como internet, etc.7 Es importante señalar que este problema se presenta en todos los niveles educativos.

En el 2009 la OMS informó que más del 50\% de los fármacos son prescritos y/o vendidos de forma incorrecta, y la otra proporción los pacientes los utilizan de forma inapropiada, esto puede deberse a la falta de políticas o estrategias para promover el uso racional de fármacos. ${ }^{8}$ De igual modo define al uso racional como al empleo correcto o apropiado de fármacos, dosis indicadas por tiempo determinado de acuerdo con las guías de práctica clínica. En cuanto al uso irracional éste puede tener diferentes patrones de uso como la automedicación y la polifarmacia que son los principales. ${ }^{9}$

En México se reportó que 80\% de la población mexicana usa medicamentos por iniciativa propia sin indicación médica y sin receta.

A nivel internacional se ha reportado una prevalencia de automedicación en estudiantes de medicina del 76.9\%. ${ }^{10}$

El personal de salud es el responsable de la prescripción, así como la promoción en el uso adecuado de los medicamentos, por lo que es necesario conocer la prevalencia e identificar las principales causas de automedicación en estudiantes universitarios del área de la salud.

\section{MATERIAL Y MÉTODOS}

Se realizó un estudio transversal; el universo del estudio fueron estudiantes universitarios del área de la salud de nivel licenciatura y postgrado, de la UNAM y del IPN, durante el ciclo escolar 20182; la unidad de observación fueron alumnos de las carreras de Licenciatura en Enfermería (LE), Licenciatura en Enfermería y Obstetricia (LEO), Licenciatura Médico Cirujano (LMC) de la UNAM e IPN, diplomado de Farmacología en Enfermería, Maestría en Enfermería (ME) y Maestría en Investigación Clínica y Experimental en Salud en el área de Farmacología Clínica (MICES), el muestreo fue no probabilístico.

Los criterios de inclusión fueron alumnos de los diferentes semestres de la LE y LEO de la Escuela Nacional de Enfermería y Obstetricia de la UNAM, Facultad de Medicina de la UNAM y del IPN, Diplomado de Enfermería de la ENEO-UNAM del nivel postgrado ME y MICES de la UNAM; criterios de 


\begin{tabular}{lc}
$\begin{array}{l}\text { Tabla 1: Características de la muestra de } \\
\text { alumnos universitarios del área de la salud. }\end{array}$ \\
\hline & Frecuencia (\%) \\
\hline Promedio de edad (DE) & $28.5(12.04)$ \\
Sexo & \\
Femenino & $218(71.5)$ \\
Masculino & $87(28.5)$ \\
Educación & \\
Licenciatura & $223(73.1)$ \\
Diplomado & $59(19.3)$ \\
Maestría & $23(7.6)$ \\
Estado civil & \\
Soltero & $293(96.1)$ \\
Casado & $7(2.3)$ \\
Divorciado & $2(0.7)$ \\
Datos perdidos & $3(1.0)$ \\
\hline
\end{tabular}

exclusión: alumnos que no quisieron participar y criterios de eliminación que no hayan respondido el cuestionario correctamente.

El cálculo de la muestra se realizó con una prevalencia de $76.9 \%$ de automedicación en estudiantes universitarios, con un nivel de confianza de 0.05 , más una tasa de no respuesta del 10\%, teniendo un tamaño de muestra de 305 estudiantes. Se incluyó a todos los alumnos del diplomado de Farmacología en Enfermería y de postgrado (78).

Se les aplicó un cuestionario sobre «Datos de automedicación», de 18 reactivos, el cual contenía datos generales como sexo, edad y 13 reactivos específicos de automedicación, se aplicó de forma individual, el tiempo de respuesta fluctuó entre 3 a 5 minutos, los datos fueron recolectados en los meses de junio y julio de 2018.

La información se capturóy analizó en Excel, se realizó un análisis descriptivo y relativo para cada variable de la investigación.

\section{RESULTADOS}

Se estudió a 305 estudiantes universitarios inscritos en los programas del área de la salud, 71.5\% correspondió al género femenino y $28.5 \%$ al masculino;
$35 \%$ tenía entre 17 y 21 años, $24 \%$ entre $22-26$ y $18 \%$ más de 42 años (Tabla 7).

Sesenta y dos punto nueve por ciento de la población consumió algún medicamento durante el último mes. En cuanto a quién le recetó el medicamento en ese lapso, 51.1\% lo hizo un médico y $16.4 \%$ se automedicó. En lo que respecta con qué bebida acostumbra a tomarse el medicamento $72.1 \%$ con agua, $11.8 \%$ con jugo y 5.6\% con infusión.

Al preguntarles qué es la automedicación los alumnos universitarios del área de la salud respondieron lo siguiente (Figura 7).

Ochenta y ocho por ciento de los alumnos del área de la salud se automedican, siendo 65\% más frecuente en las mujeres observándose una diferencia estadísticamente significativa con un $\mathrm{p}=0.009 ; 13.7 \%$ lo hace de forma frecuentemente y el $2.9 \%$ casi siempre (Figura 2 ). La razón principal por lo que prefirió automedicarse es por la falta de tiempo con un 42.1\%, 37.4\% lo hace por la accesibilidad a los medicamentos y $14.4 \%$ por problemas de economía (Figura 3). El principal lugar donde adquieren los medicamentos es en la farmacia con un $64.6 \%$, reserva en casa $46.4 \%$, supermercado $9.2 \%$ y $5.6 \%$ lo obtiene con un familiar de un hospital.

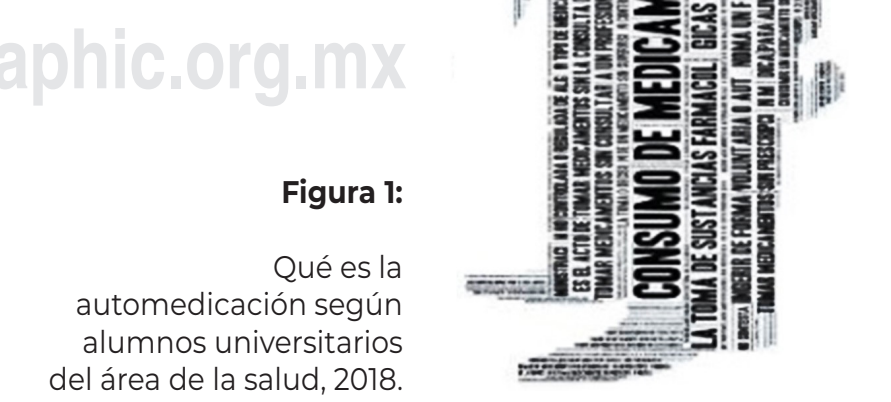


El motivo de salud por el que se automedican los alumnos es por algias con un 73.8\% y $49.7 \%$ por síntomas de gripe, $33.8 \%$ por fiebre y $27.8 \%$ por gastroenteritis (Figura 4). Derivado de la automedicación 3.6\% ha tenido algún evento adverso por la automedicación como alergia, shock anafiláctico, irritación, sangrado e hipersensibilidad, eritemas, braquipnea, náuseas, mareos, estreñimiento (Figura 5).

El patrón de automedicación está encabezado por analgésicos con 28.2\%, antinflamatorios 10\% y antibióticos 7.2\% (Figura 6).

Los estudiantes del área de la salud que más se automedican es enfermería con una RP $=2.17$.

\section{DISCUSIÓN}

La automedicación es una conducta frecuente en estudiantes del área de la salud. La prevalencia fue mayor (88\%) con respecto a la población mexicana (80\%) y en estudiantes de medicina (76.9\%) y fue muy similar a los universitarios venezolanos quienes tienen una prevalencia de $87 \%,{ }^{1}$ así como en la población general del mismo país y la de Chile y Colombia;1-13 lo cual podría comprometer su salud,

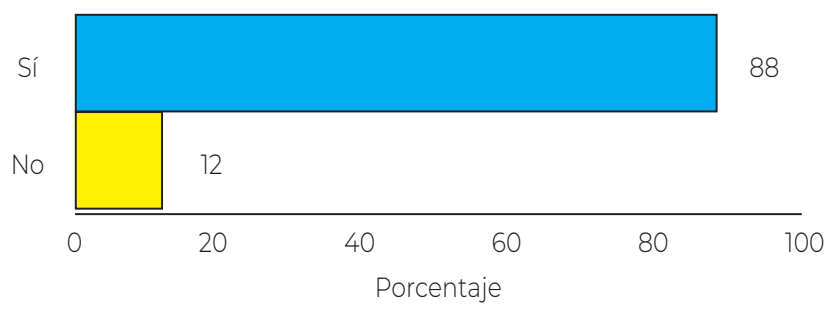

Figura 2: Automedicación de los estudiantes universitarios del área de la salud, 2018.

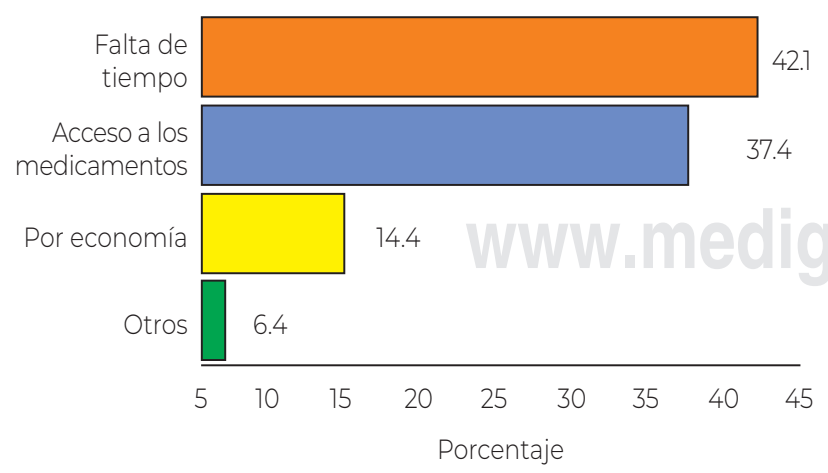

Figura 3: Causas por las que se automedican los estudiantes del área de la salud, 2018.

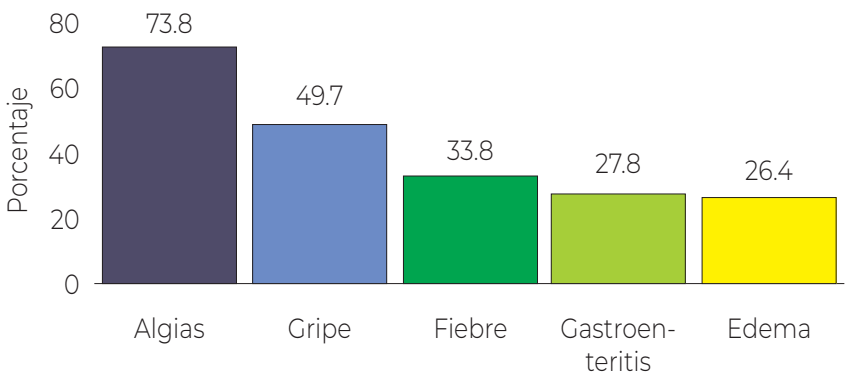

Figura 4: Motivo de salud por qué se automedican de los estudiantes del área de la salud, 2018.

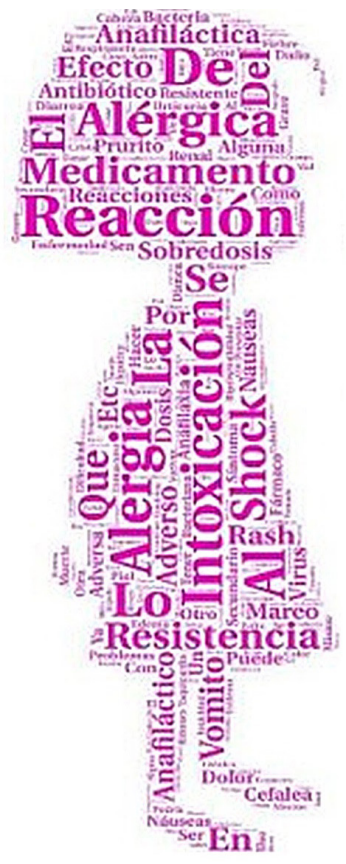

la de su familia, comunidad y de la población en general.

Existen diversos factores que influyen para que la población se automedique, uno de los principales factores sociales son: la presión que ejerce un grupo o familiares de ofrecer una alternativa de solución a los problemas de salud-enfermedad; la falta de tiempo para acudir a la consulta médica principalmente porque los tiempos de espera son largos o bien porque los servicios de atención son lentos; así como también una sociedad dominada por las obligaciones académicas, laborales aunadas a las actividades domésticas. 


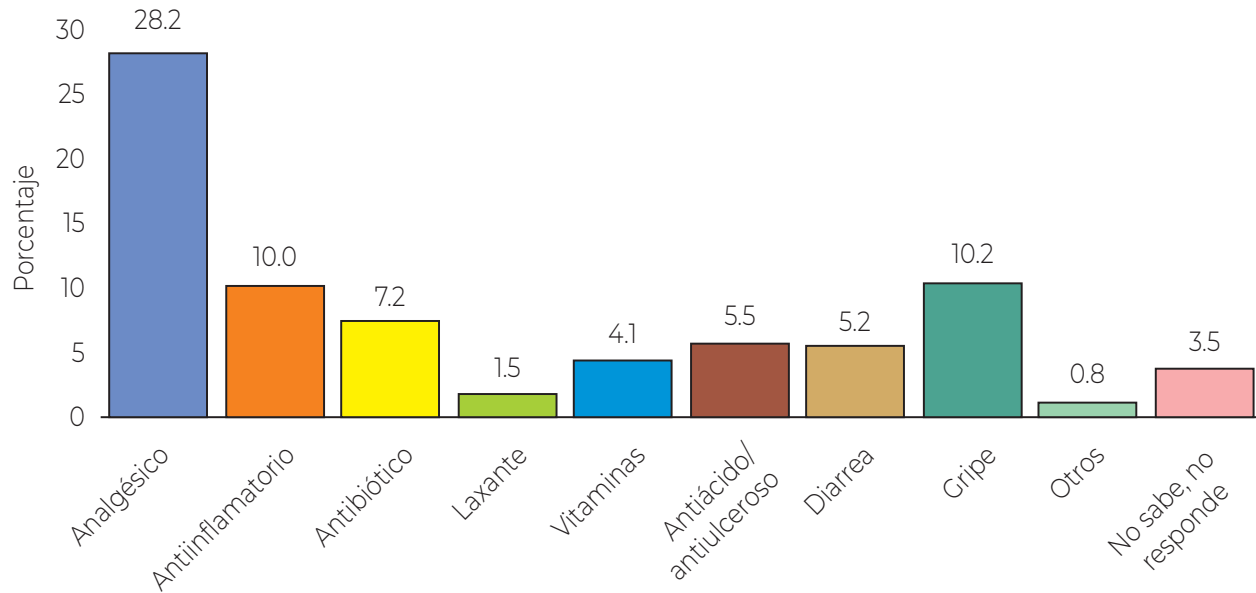

\section{Figura 6:}

Patrón de uso de fármacos de automedicación de los estudiantes universitarios del área de la salud, 2018.
Otro de los factores son los económicos derivados del desempleo, las malas condiciones de vida $y$, sobre todo, el bajo ingreso económico familiar que impide acceder a los servicios de salud. Así mismo, French en 1997 planteó que la decisión de la automedicación tiene que ver también con factores culturales, como: la falta de acceso a los servicios de salud y la escasa educación sanitaria, lo que ha permitido a la industria farmacéutica ganar espacios para difundir información sesgada de los medicamentos, así como invitar a la población a consumir fármacos poco seguros. Es importante señalar que la publicidad puede influir en los profesionales de la salud, ya que en ocasiones se prescriben antibióticos de amplio espectro para tratar infecciones simples, los cuales, además, son más costosos. Otro factor que ha influido es la pérdida de credibilidad sanitaria basada en el deterioro de la relación médico-paciente, enfermera-paciente, por lo que hoy en día se confía más en los medios de comunicación que en el personal de salud.

En el caso de los pacientes también la publicidad influye para que se automediquen, por otro lado, los pacientes asumen diversos comportamientos, como hay quienes utilizan los sobrantes de tratamientos anteriores o de otros, sin consultar al médico. Algunos, simplemente usan medicamentos sin prescripción médica, otros usan medicamentos que necesitan receta y aun así, los obtienen sin ésta. Cabe señalar que los que se automedican generalmente desconocen la gravedad de usar fármacos indiscriminadamente y hay otros quienes simplemente mencionan que no existe ninguna sustancia química inocua.
El principal motivo del porqué se automedica la población universitaria del área de la salud en México es muy similar a la población de Brasil, ya que en ambos países se automedican por dolor. 3,1,14,15

Noventa y siete por ciento de las mujeres utilizan medicamentos, $64.6 \%$ para cefaleas y migrañas, 30\% en dolores musculares y $23.2 \%$ para molestias estomacales y odontológicas.

Respecto a los eventos adversos asociados con la automedicación se reportó una prevalencia de $3.6 \%$, la cual es menor con respecto a la comunidad universitaria de Venezuela (71\%); quizás esta diferencia se deba al universo de estudio, ya que en este estudio fueron alumnos universitarios del área de la salud y en el caso de la comunidad universitaria de Venezuela la población de estudio fueron alumnos de las especialidades de Educación Ambiental. Los eventos que se presentaron con más frecuencia en la comunidad universitaria de México son: alergia, shock anafiláctico, irritación, sangrado e hipersensibilidad, eritemas braquipnea, náuseas, mareos, estreñimiento; mientras que en la comunidad universitaria de Venezuela fueron: náuseas y dolores estomacales, somnolencia, cefalea y taquicardia. Es importante señalar que $42.5 \%$ de los(as) jóvenes sólo reconocen como eventos adversos de fármacos a la somnolencia y taquicardia, quizá porque los otros síntomas son generales e inespecíficos. Existen estudios donde nos indican que a mayor nivel cultural mayor conciencia para reconocer los peligros de los fármacos.

Es importante señalar que la automedicación de productos de venta con receta es principalmente 
un problema en los países en vías de desarrollo, en los que las farmacias, así como en comercios no autorizados, venden los medicamentos sin exigir la receta. Hardor observó en Filipinas que la gente conserva copias de las prescripciones médicas para volver a usarlas, asimismo, refirió que las consultas médicas son caras y usar repetidamente una receta es una forma de economizar, usando los medicamentos disponibles, sin percatarse en ocasiones de la fecha de caducidad.

Aunado a lo anterior, existe hoy en día la venta libre de medicamentos por internet, lo cual permite comprar medicamentos que por ley en su país sólo se venden con receta médica.

Actualmente, el internet es una fuente de información y una herramienta de trabajo para los profesionales de la salud, pero también es un problema de seguridad del paciente cuando la usan, ${ }^{16}$ ya que a través de las TICs hay sitios donde se puede compartir información sobre salud que pueden contribuir a modificar su estilo de vida, pero también se puede encontrar información errónea y sesgada, por conflicto de intereses. ${ }^{17}$

La razón principal por la que los alumnos del área de la salud se automedicaron fue: la falta de tiempo con un $42.1 \%$, este dato concuerda con el estudio realizado entre estudiantes universitarios de enfermería de la Universidad Federal de Goiás, Brasil, (50\%) ${ }^{18}$ y con el estudio de docentes en enfermería. ${ }^{19}$ Los motivos asociados a éste es la falta de acceso a los servicios de salud, largos tiempos de espera en la atención médica, desinterés por el tratamiento médico debido a la falta de éxito de tratamientos anteriores y los conocimientos propios del área de la salud que ayudan en la elección del medicamento. ${ }^{20}$ Así como el fácil acceso y en lo que respecta a la parte económica es más barato comprar en la farmacia en lugar de primero solicitar la consulta médica. ${ }^{18}$

\section{CONCLUSIONES}

El conocer por qué se automedican los alumnos del área de la salud y qué medicamentos usan, es un paso fundamental para tener un patrón de uso de medicamentos con el menor riesgo posible.

La automedicación es frecuente entre los estudiantes universitarios del área de la salud, su prevalencia es mayor a la nacional y muy similar a estudios realizados en estudiantes universitarios de otros países.

Quizás los estudiantes se automedicaron con base a su propia información, así como también por sugerencias de familiares y la principal causa que los motivó a realizar esta práctica fue la falta de tiempo para ir al médico.

Cabe señalar que los medicamentos utilizados por los estudiantes del área de la salud no necesitan prescripción; sin embargo, su consumo irracional puede causar eventos adversos. Por lo que el personal de salud debe establecer estrategias de prevención de uso racional de medicamentos con el propósito de evitar el enmascaramiento de signos y síntomas, agravar el proceso de la enfermedad, resistencia a medicamentos, intoxicación e inclusive la muerte. Asimismo, se debe de fomentar que la automedicación es un componente del autocuidado; por lo que se debe incentivar el uso racional de medicamentos, informar sobre los fármacos y las complicaciones que pueden originarse por el uso irracional y promover la automedicación responsable. ${ }^{18}$

\section{AGRADECIMIENTOS}

A los estudiantes del área de la salud de nivel licenciatura y postgrado por el apoyo en la recolección de información que con su amable colaboración se logró que esta investigación fuera posible.

\section{BiBLIOGRAFÍA}

1. Baos V. Estrategias para reducir los riesgos de la automedicación. Información Terapéutica Sistema Nacional de Salud. 2000; 24: 147-152.

2. Jain S, Malvi R, Purviya JK. Concept of self medication: a review. Int J Pharm Biol Arch. 2011; 2 (3): 831-836.

3. Sánchez C, Nava M. Análisis de la automedicación como problema de salud. Enf Neurol (Mex). 2012; 11 (3): 159-162.

4. Ruiz E. Risks of self-medication practices. Curr Drug Saf. 2010; 5 (4): 315-323.

5. Baños JE, Bosch R, Toranzo I. La automedicación con analgésicos. Estudio en el dolor odontológico. Med Clin (Barc). 1991; 96: 248-251.

6. Zyl-Schalekamp CV. Self-medication in three orange free state communities. S Afr Med J. 1993; 83: 345-346.

7. López-Cabra CA, Cálvez-Bermúdez JM, DomínguezDomínguez C, Urbina-Bonilla AP, Calderón-Ospina CA, Vallejos-Narváez Á. Automedicación en estudiantes de medicina de la Universidad del Rosario en Bogotá D. C., 
Colombia. Rev Colomb Cienc Quím Farm. 2016[Acceso: 18 de enero de 2019]; 45 (3): 374-384. Disponible en: https://revistas.unal.edu.co/index.php/rccquifa/article/ view/62018/59438

8. World Health Organization. Medicines use in primary care in developing and transitional countries: fact book summarizing results from studies reported between 1990 and 2006. Bull World Health Organ; 2009; 87 (10): 804.

9. Salazar J, Bello L, Toledo A, Añez R, González L, Rojas J et al. Comportamiento epidemiológico de la automedicación y polifarmacia en individuos adultos del sector Dalia de Fernández, Municipio San Francisco. AVFT [Internet]. 2013; 32 (1): 1-15. Recuperado de: https://www.redalyc.org/artículo. oa?id=55931088001

10. Sawalha AF. A descriptive study of self-medication practices among Palestinian medical and nonmedical university students. Res Social Adm Pharm. 2008; 4 (2): 164-172.

11. De Pablo MM. La automedicación en la cultura universitaria. Rev de Investigación. 2011; 35 (73): 219-239. [Recuperado 18 de enero de 2019] Disponible en: https://www.redalyc.org/ comocitar.oa?id=376140388010

12. Moya S. Estudio - Prevalencia de automedicación en consultantes a un centro de atención ambulatorio adosado a un hospital de una Comuna de Santiago [Tesis de grado presentada como parte de los requisitos para optar al Título de Químico Farmacéutico, Memoria para optar al título de química farmacéutica]. Santiago de Chile: 2012. [Recuperado 18 de enero de 2019] Disponible en: http://repositorio.uchile.cl/handle/2250/111220

13. Lopez JJ, Dennis R, Moscoso SM. Estudio sobre la Automedicación en una Localidad de Bogotá. Rev Salud Pública. 2009; 11 (3): 432-444.
14. Derly-Damasceno D, de Souza-Terra F, Vieira-Zanetti HH, Dias-D'Andréa E, Ribeiro-da-Silva HL, Leite JA. Automedicação entre graduandos de enfermagem, farmácia e odontologia da universidade federal de alfenas. Rev Min Enf. $2007 ; 2$ (7): 48-52.

15. Guillem-Sáiz P, Francès-Bozal F, Gimenez-Fernández F, Sáiz-Sánchez C. Estudio sobre automedicación en población Universitaria Española. Rev Clin Med Fam. 2010; 3 (2): 99-103.

16. Solé-Balcells FJ. Internet en medicina ¿Una ayuda, un problema? Actas Urol Esp [Internet]. 2003 [citado 2019 Ene 18]; 2003; 27 (3): 180-184. Disponible en: http:// scielo.isciii.es/scielo.php?script=sci_arttext\&pid=S021048062003000300002\&ling=es.

17. Scanfeld D, Scanfeld V, Larson EL. Dissemination of health information through social networks: twitter and antibiotics. Am J Infect Control. 2010; 38 (3): 182-188.

18. Ferreira-Souza LA, Damázio da Silva C, Carvalho-Ferraz G, Faleiros-Sousa FAE, Varanda-Pereira L. Prevalencia y caracterización de la práctica de automedicación para alivio del dolor entre estudiantes universitarios de enfermería. Rev Latino-Am Enfermagem [Internet]. 2011; 19 (2). [Recuperado 3 de diciembre de 2018] Disponible en: http://www.scielo.br/pdf/rlae/v19n2/es_04.pdf

19. Cerqueira CS, Diniz MFFM, Lucena GT, Dantas AF, Lime GMB. Perfil da automedicação em acadêmicos de enfermagem na cidade de João Pessoa. Conceitos. Rev Bras Farm. 2011; 92 (3): 186-190.

20. Zafar SN, Syed R, Waqar S, Zubairi AJ, Vagar T et al. Selfmedication amongst university students of Karachi: prevalence, knowledge and attitudes. J Pak Med Assoc. 2008; 58 (4): 214-217. 